\title{
Validation of a New Dynamic Muscle Fatigue Model and DMET Analysis
}

\author{
Deep Seth ${ }^{1}$, Damien Chablat ${ }^{1}$, Fouad Bennis ${ }^{1}$, Sophie Sakka ${ }^{1}$, Marc Jubeau ${ }^{2}$ and Antoine Nordez ${ }^{2}$ \\ ${ }^{1}$ IRCCyN, Ecole Centrale de Nantes, France \\ 2 STAPS, Université de Nantes, France
}

\begin{abstract}
Automation in industries reduced the human effort, but still there are many manual tasks in industries which lead to musculo-skeletal disorder (MSD). Muscle fatigue is one of the reasons leading to MSD. The objective of this article is to experimentally validate a new dynamic muscle fatigue model taking cocontraction factor into consideration using electromyography (EMG) and Maximum voluntary contraction (MVC) data. A new model (Seth's model) is developed by introducing a co-contraction factor ' $n$ ' in $\mathbf{R}$. Ma's dynamic muscle fatigue model. The experimental data of ten subjects are used to analyze the muscle activities and muscle fatigue during extension-flexion motion of the arm on a constant absolute value of the external load. The findings for co-contraction factor shows that the fatigue increases when co-contraction index decreases. The dynamic muscle fatigue model is validated using the MVC data, fatigue rate and co-contraction factor of the subjects. It has been found that with the increase in muscle fatigue, co-contraction index decreases and $90 \%$ of the subjects followed the exponential function predicted by fatigue model. The model is compared with other models on the basis of dynamic maximum endurance time (DMET). The co-contraction has significant effect on the muscle fatigue model and DMET. With the introduction of co-contraction factor DMET decreases by $25.9 \%$ as compare to R. Ma's Model.
\end{abstract}

Index Terms - Muscle fatigue, maximum voluntary contraction (MVC), muscle fatigue model, co-contraction, fatigue rate, electromyography (EMG), maximum endurance time (MET), industrial ergonomics.

\section{INTRODUCTION}

In the field of industrial bio-mechanics, muscle fatigue is defined as "any exercise-induced reduction in the maximal capacity to generate the force and power output" (Vøllestad 1997). In industries, mostly repetitive manual tasks leads

\footnotetext{
E-mail: Deep.Seth@irccyn.ec-nantes.fr, Damien. Chablateirccyn.ec-nantes.fr,

Fouad.Bennisdec-nantes.fr,

Sophie.Sakka@irccyn.ec-nantes.fr,

Marc. Jubeau@univ-nantes.fr,

Antoine.Nordez@univ-nantes.fr
}

to work related Musculo-Skeletal Disorder (MSD) problems (Nur, Dawal, and Dahari 2014; Punnett and Wegman 2004). Work on repetitive and uncomfortable tasks can be painful (Huppe, Muller, and Raspe 2006) and leads to MSD (Chaffin, Andersson, and Martin. 1999; WorldHealth-Organization 2003). One of the reasons for MSD can be muscle fatigue (Nur, Dawal, and Dahari 2014). To limit MSD, the study of muscle fatigue can be very important. There are various factors which contribute to MSD problems. These factors are repetitive tasks, excessive efforts, long duration static tasks, uncomfortable working conditions, etc. These factors can cause problems like rapid muscle fatigue, increase in recovery time, muscles tension, muscle pain, muscle injury, tendon injury, nerves injury, etc. Muscle fatigue can have significant effect on muscle endurance. Muscle endurance is the ability to do some work or task over and over for an extended period of time without getting tired. The time when the force production can no longer be maintained is defined as the endurance time (ET). In this study we are mainly focusing on muscle fatigue during dynamic repetitive tasks.

Various static and dynamic muscle fatigue models were proposed earlier to study muscle fatigue (Ding, Wexler, and Binder-Macleod 2003; Hill 1938; Ma et al. 2008; Syuzev, Gouskov, and Galiamova 2010; Xia and Lawa 2008). Silva (Silva, Pereira, and Martins 2011) simulate the hill's model and validate it theoretically using Opensim. Dynamic model proposed by L. Ma (Ma et al. 2008) and R. $\mathrm{Ma}(\mathrm{Ma}$ et al. 2011) have experimented validation for fatigue in arm with a static drilling posture and dynamic push-pull operation respectively. Maximum endurance time (MET) of muscle shows capacity of muscle to generate force up to the point of initiation of muscle injury and MSD. L. Ma and R. Ma also determined the maximum endurance time (MET) for static and dynamic condition respectively. Some other dynamic fatigue models were also introduced by various authors (Freund and Takala 2001; Liu, Brown, and Yue 2002; $\mathrm{Ma}$, Chablat, and Bennis 2012a), however, no consideration about the co-contraction of paired muscles is taken into account in these models. The study of Missenard (Missenard, Mottet, and Perrey 2008) is one of the example to understand the fatigue and co-contraction, which shows the reduction in accuracy of tasks with fatigue. The main objective of this study is to revise the dynamic muscle fatigue model pro- 
posed by R. Ma by including the co-contraction factor of paired muscles into the model and experimentally validating the muscle fatigue model. The experiment duration part of each subject will then be compared to the dynamic maximum endurace time (DMET) determined for our model. The determined DMET will also be compared with L. Ma's and R. Ma's model. In this article, we focus on the study of muscle co-contraction activity, using elbow joint muscle groups as a target. Experiments were performed on 10 subjects to study the EMG activity of bicepss, tricepss and trapezius muscles. Processing of raw EMG data (Doguet and Jubeau 2014; Guvel et al. 2011) was done. With the assistance of EMG, the function of co-contraction is confirmed and calculated. The comparison of proposed model with L. Ma's model and R. Ma's models on the basis of DMET is also done. The comparison show the significant advantage of our proposed model (which will later called in article as Seth's model) over other models. The work related to the proposed model to limit musculoskeletal disorder is presented in VRIC 2016, France (Seth et al. 2016).

\section{Proposed dynamic model of MUSCULAR FATIGUE}

The dynamic muscle fatigue model is applicable on the dynamic motion of the human body parts. A dynamic muscle fatigue model was proposed by L. Ma (Ma et al. 2008; Ma et al. 2009) firstly applied on static drilling task. R. Ma (Ma et al. 2011; Ma, Chablat, and Bennis 2012b) develops this model for the dynamic motions like push/pull operation of the arm. However, the co-contraction of the muscles are not included in both models. In dynamic muscle fatigue model (Ma, Chablat, and Bennis 2012a), we selected two parameters $\Gamma_{\text {joint }}$ and $\Gamma_{M V C}$ to build our muscle fatigue model. The hypotheses can then be incorporated into a mathematical model of muscle fatigue which is expressed as follows:

$$
\frac{d \Gamma_{c e m}(t)}{d t}=-k \cdot n \cdot \frac{\Gamma_{c e m}}{\Gamma_{M V C}} \Gamma_{j o i n t}(t)
$$

where, $k$ is the fatigue factor (defines the rate of fatigue) and $n$ is the co-contraction factor.

And, if $\Gamma_{\text {Joint }}$ and $\Gamma_{M V C}$ hold constant, the model can then simplify as follows:

$$
\begin{gathered}
\Gamma_{c e m}(t)=\Gamma_{M V C} \cdot \mathrm{e}^{-k \cdot n \cdot C t}, \quad\left\{\text { where }, \quad C=\frac{\Gamma_{\text {Joint }}}{\Gamma_{M V C}}\right\} \\
k=\frac{-1}{n \cdot C t} \cdot \ln \left(\frac{\Gamma_{c e m}(t)}{\Gamma_{M V C}}\right)
\end{gathered}
$$

The other parameters for this model are same as in Table $1 . n$ is the co-contraction factor.

\section{Dynamic Maximum Endurance Time (DMET)}

Endurance (also related to sufferance, resilience, constitution, fortitude, and hardiness) is the ability of a person or muscle to exert itself and remain active for a longer period of time, as well as its ability to resist, withstand, recover from, and have immunity to trauma, wounds, or fatigue. It is

\begin{tabular}{|l|l|l|}
\hline Elements & Unit & Description \\
\hline \hline$k$ & $\min ^{-1}$ & Fatigue factor, constant \\
$\Gamma_{M V C}$ & N.m & Maximum torque on joint \\
$\Gamma_{\text {Joint }}$ & N.m & Torque from external load \\
$\Gamma_{\text {cem }}$ & N.m & Current capacity of the muscle \\
\hline
\end{tabular}

Table 1: Parameters of dynamic muscle fatigue model

usually used in aerobic or anaerobic exercise. The definition of 'long' varies according to the type of exertion minutes for high intensity anaerobic exercises, hours or days for low intensity aerobic exercise.

For better understanding the DMET we can see the Fig. 1 which illustrates R. Ma's assessment of DMET from the fatigue model. The red curve in the figure represents the reduction in the muscle capacity or strength with respect to time. The blue cycles represent the dynamic repetitive task and the straight blue line parallel to $x$ axis represent the external load. The black dot lines represent the point after which there is a chance of MSD. R. Ma's model show DMET approached model but as we have included cocontraction in our model DMET will be lesser for our model as compared to R. Ma's Model.

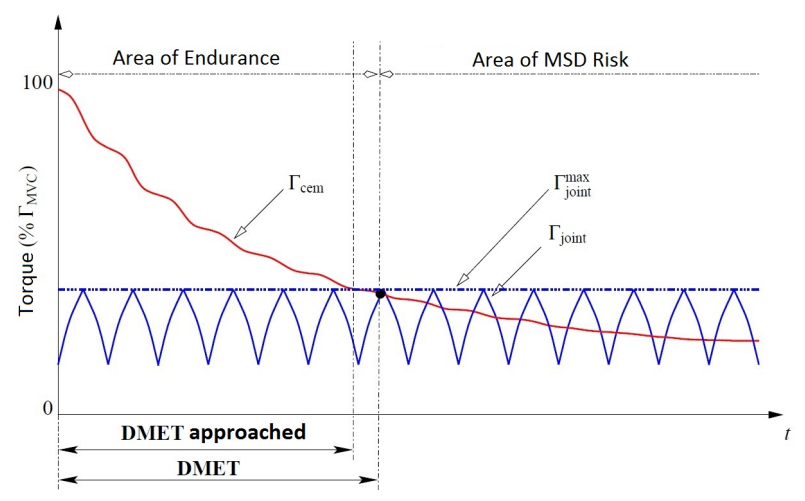

Figure 1: The endurance time for dynamic conditions (Ma 2012)

The reduction in the maximum exertable force or torque capacity of muscle is one of the hypothesis for the proposed dynamic muscle fatigue model. Maximum endurance time (MET) represents the maximum time during which a static load can be maintained (Ahrache, Imbeau, and Farbos 2006). The MET is generally calculated as the percentage of the maximum voluntary contraction (\%MVC) or to the relative force/torque $\left(\Gamma_{M V C}=\% \mathrm{MVC} / 100\right)$. MET models are used to predict endurance time of a muscle under static or dynamic conditions.

By solving Eq. 2 for $\Gamma_{c e m}(T)=\Gamma_{\text {Joint }}^{\max }$ with physical and mechanical parameters of motion using the method described by R. Ma (Ma 2012), DMET can be rewritten as:

$$
D M E T=-\frac{1}{n \cdot d \cdot k} \cdot \frac{\ln \left(f_{M V C}\right)}{f_{M V C}}, \text { with } 0<d \leq 1
$$


Here, $f_{M V C}=\frac{\Gamma_{\text {joint }}^{\max }}{\Gamma_{M V C}}$. The parameter ' $d$ ' involved in the DMET model varies between 0 and 1 and depends on the magnitude and speed of the movement. ' $d$ ' closer to 0 represents dynamic conditions and ' $d$ ' closer to 1 represents static conditions.

\section{Methodology AND DATA Processing}

\section{Push-Pull Operation and Muscles activities}

The push/pull motion of the arm is flexion and extension of the arm about the elbow. The Push/pull activities with the muscle activation is shown in Fig. 2. There is co-contraction between Push and pull phases, however when phase changes from pull to push we can observe there is delay. The muscle activities shown in Fig. ?? is for flexion and extension in saggital vertical plane.

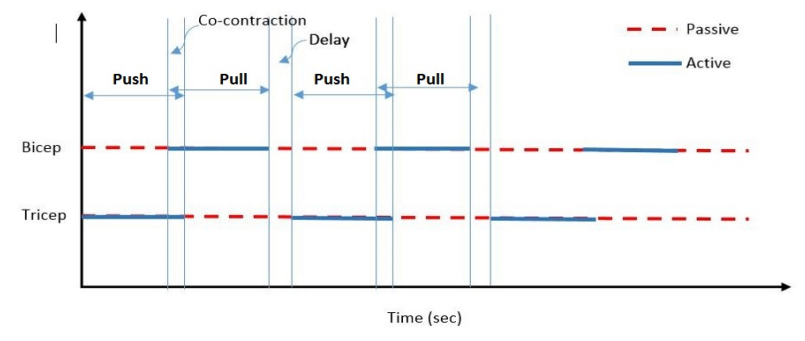

Figure 2: Push/Pull Motion and Muscles activities

\section{Co-contraction factor ' $n$ '}

The co-contraction is the simultaneous contraction of both the agonist and antagonist muscle around a joint to hold a stable position at a time. Assumptions made for finding cocontraction factor, which depict that, the co-contraction is the common intersecting area between the two groups of muscles, see the yellow area in Fig. 3. The co-contraction factor will be the same for each agonist and antagonist activities.

The co-contraction area can be understand by the Fig. 3 . This figure is just an example representation of the muscle activity during one motion cycle. In this figure, we can see the common EMG activity between biceps and triceps muscle shown by the orange color, which is co-contraction area. The formula for calculating the co-contraction index $C_{A}$ (represent part of co-contraction for each cycle) from EMG activities is given in Eq. 5. The trapezius activity shown along with the two muscles is co-activation.

$$
\begin{gathered}
C_{A}=\frac{\int_{t_{0}}^{t_{100}} E M G_{\text {commom }} \times d t}{\int_{t_{0}}^{t_{100}}\left[E M G_{\text {agonist }}+E M G_{\text {antagonist }}\right] \times d t} \\
n=1+C_{A}
\end{gathered}
$$

Where, $E M G_{\text {common }}$ is the common area share by the EMG activity of bicepss and tricepss, EMG agonist and
$E M G_{\text {antagonist }}$ are the full activities of the biceps and tricepss muscle.

The co-contraction index $C_{A}$ can also be represented as follows:

$C_{A}=$ co-contraction index between the two group of muscles.

$$
C_{A}=a \cdot \exp b \cdot x
$$

Where, $a$ and $b$ are constant float integers and $x$ is the time.

The activities of both muscles are normalized with respect to the normalized value of the activities of each muscle, calculated using Eq. 8 described in section .

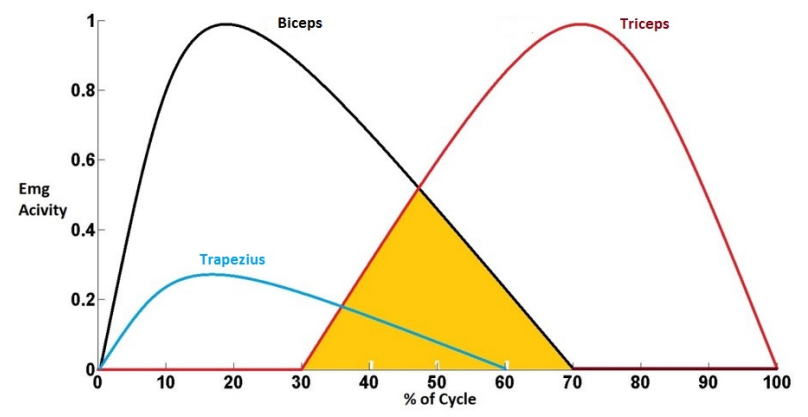

Figure 3: A representative plot of EMG activity of biceps, tricepss and trapezius normalized with the maximum value of each muscles activity for one cycle

\section{Subjects description}

Ten male subjects participated in the experiments. The subjects details are given in Tab. 2. All the subjects were sportive.

\begin{tabular}{|l|l|l|c|c|c|c|}
\hline subject & Age & Weight $(\mathbf{k g})$ & Height $(\mathbf{c m})$ & Upper Arm (cm) & Forearm $(\mathbf{c m})$ & Sport \\
\hline \hline 1 & 28 & 89 & 185 & 29 & 26.5 & Running \\
2 & 24 & 80.2 & 183.5 & 31.5 & 28 & Gym \\
3 & 20 & 69.8 & 180.1 & 30 & 29.5 & Handball \\
4 & 20 & 80.9 & 177 & 29.8 & 29 & Handball \\
5 & 21 & 62.2 & 172.8 & 29.2 & 26.5 & Tennis \\
6 & 25 & 61.1 & 164.8 & 26 & 24.5 & Rugby \\
7 & 26 & 74 & 176 & 28.5 & 27 & Tennis \\
8 & 27 & 66 & 181 & 29.5 & 26.5 & wall climb \\
9 & 23 & 66.3 & 164 & 27 & 25.5 & Swimming \\
10 & 26 & 85 & 184 & 29 & 26.5 & Football \\
\hline
\end{tabular}

Table 2: Subjects anthropometric data and description

\section{Experiment Protocol}

A biodex (REF) system is used to perform flexion and extension in isotonic mode in vertical plane as in Fig. 4, with $70^{\circ}$ range of motion $\left(-20^{\circ}\right.$ to $\left.50^{\circ}\right)$. Each protocol lasts 1 minute which includes 20 cycles (flexion + extension). The test protocol repetition continues till exhaustion of the subjects. During the fatigue test protocol, the external load will be $20 \%$ of MVC. MVC is tested every one minute and at the end of the protocol. To restrict the backward motion of the arm, a support is provided behind the upper arm. 


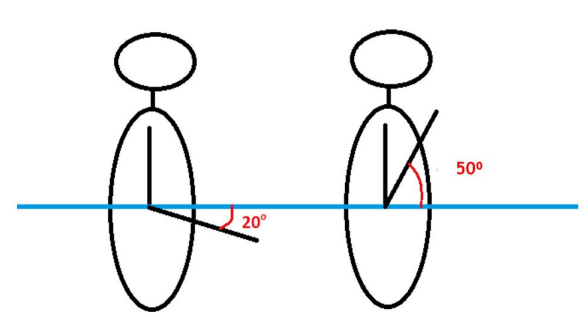

Figure 4: Arm movement range while flexion and extension in vertical plane

\section{Data Acquisition}

A Biodex system 3 research (Biodex medical, shirley, NY) isokinetic dynamo-meter is used to measure the value of the elbow angle, velocity and torque. The Electromyographic surface electrodes (Ag/Agcl, 4mm diameter) fixed parallel to bicepss brachii, triceps and Trapezius muscles to record their electrical activities at $2000 \mathrm{~Hz}$ frequency. Powerlab data acquisition system is used to record all the experimental data. The experiments were performed in laboratory STAPS, University of Nantes, France.

\section{Data Processing and analysis}

All the raw data were processed using standardized MATLAB program. Data processing includes noise filtering from raw EMG data with the band pass filter (butterworth, 2nd order, 10-400 Hz) and normalization of the data. EMG was normalized with a value calculated by Eq. 8. The total number of cycles compared for all the ten subjects are 1998 cycles. All the cycles are normalized on time scale and compared. The cycle selection for the flexion and extension phases is done according to the velocity change in each cycle. The collective EMG plots for bicepss, tricepss and Trapezius muscles are show in Fig. 5 and Fig. 6 for all the ten subjects and the collective comparison for the mechanical data position, velocity and torque is shown in Fig. 7 and Fig. 8 for all the ten subjects.

Curves representation in Figs. 5, 6, 7and 8 are as follows:

--_ Blue color curves show mean EMG activity.

I Red bar plotted on blue curves show the standard deviation of all the EMG activities along the mean.

- Black dotted curves show the maximum and minimum reach from the EMG activies. All the cycles are normalized according to the equation:

$$
\text { value }_{\text {Normalization }}=\text { value }_{\text {std }} \max +3 \sigma
$$

value $_{\text {Normalization }}$ : Under it all the muscle activity will be normalized.

value $_{\text {std }}^{\max }$ : Maximum value of standard deviation along the mean.

$\sigma:$ Standard Deviation

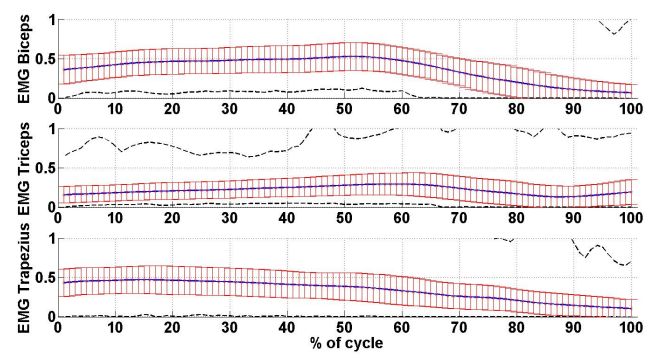

Figure 5: Flexion phase: Mean and Standard deviation plots for EMG data of bicepss, tricepss and trapezius, all subjects

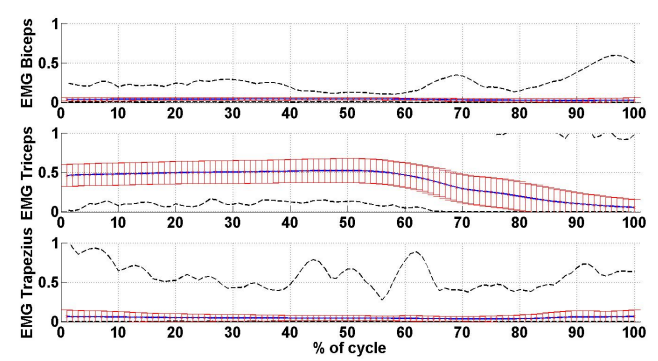

Figure 6: Extension phase: Mean and Standard deviation plots for EMG data of bicepss, tricepss and Trapezius, all subjects

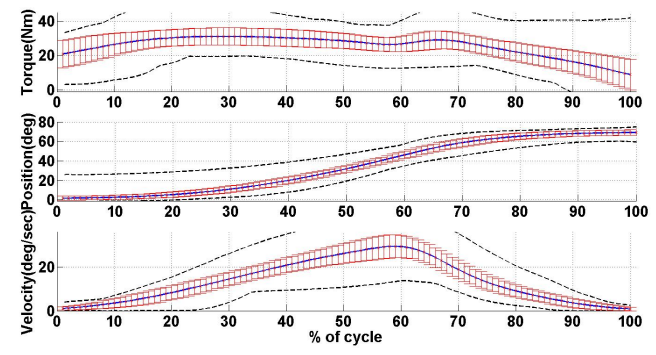

Figure 7: Mean and Standard deviation plots for velocity, position and torque in flexion Phase for all subjects

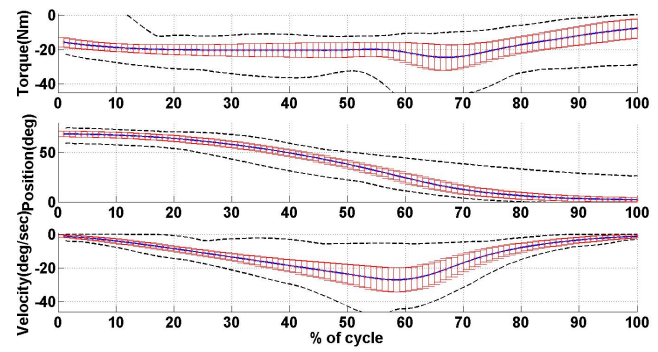

Figure 8: Mean and Standard deviation plots for velocity, position and torque in extension Phase for all subjects 


\section{RESULTS AND DISCUSSION}

The raw data obtained after the fatigue test is processed and the results are discussed in this section. After processing the EMG data of all the muscle groups from Figs. 5, 6, 7 and 8, we can observe that when the bicepss are active during the flexion phase. There are always some activities from the tricepss and on the other hand when tricepss are active during pull phase, the bicepss are almost passive or activities are very close to zero. We can also observe the co-activation of trapezius muscle with the activation of bicepss. The activation of tricepss with the bicepss is co-contraction between two muscles during the flexion phase.The co-activation of the trapezius muscle is observed mostly in the flexion phase.

The co-contraction index calculated by using Eq. 5 is fitted with Eq. 7 (looks almost linear) described in section III. The Figs. 9 - 18 show the fitted graphs for the co-contraction percentage for test cycles of all ten subjects. In Figs. 9 18 blue dots show the percentage area of contraction during each extension-flexion cycle and red curves show the exponential fit for the percentage co-contraction. This shows that the co-contraction percentage for activity between the muscles reduce as the fatigue test proceeds or the muscles get fatigued. By Eqs. 3 and 5 we can find $n_{i}$ as shown in Tab. 3, where $i$ is the subject number.

We can notice that only the subject number 8 in Fig. 16 has increasing slope for the co-contraction area, this behavior can be associated with his sport activity which is rock climbing and very different from other subjects see Tab. 2.

The MVC values are measured between each protocol of one minute. We can see in most of the cases MVC decreases as fatigue increases. The MVC is same as $\Gamma_{\text {cem }}$ used in our model. The theoretical and experimental evolution of $\Gamma_{\text {cem }}$ is on the basis of $k$ (fatigue rate) using Eqs. 2 and 3 and calculated, $n_{i}$ and $C=0.2$. The evolution of $\Gamma_{c e m}$ extension for fatigue parameter ' $k$ ' is shown in Figs. 19, 21, 23, 25, 27, 29, 31, 33, 35 and 37. Similarly the evolution of $\Gamma_{\text {cem }}$ flexion is shown in Figs. 20, 22, 24, 26, 28, 30, 32, 34, 36 and 38. In these figures blue lines show the MVC measured for flexion and extension after each test protocol of 1 minute. The MVC values measured are $\Gamma_{c e m}(t)$, used in calculating fatigue rate ' $k$ ' using Eq. 3. The theoretical $\Gamma_{c e m}$ is then calculated w.r.t minimum, maximum and average value of fatigue rate using Eq. 2. The theoretical and experimental evolutions of $\Gamma_{\text {cem }}$ show that the experimental values are well fit with in the theoretical model. The co-contraction factor have significant effect on the model. The minimum, maximum and average value of ' $k$ ' for each subject are shown in Tab. 4 . The red, pink and black dotted curves in Figs. 19 - 38 represent theoretical $\Gamma_{c e m}$, calculated from minimum, maximum and average values of fatigue rate ' $k$ ' respectively. The experimentally calculated values of $\Gamma_{c e m}(t)$ is mostly in the range of theoretical $\Gamma_{c e m}$, which validates our muscle fatigue model. The fatigue rate increased with the input of co-contraction factor in the fatigue model, which shows the significant effect of co-contraction factor in the fatigue model. In Tab. 3 and $4, i$ represents subjects number.

\begin{tabular}{|c|c|c|c|c|c|c|c|c|c|c|}
\hline$i$ & $\mathbf{1}$ & $\mathbf{2}$ & $\mathbf{3}$ & $\mathbf{4}$ & $\mathbf{5}$ & $\mathbf{6}$ & $\mathbf{7}$ & $\mathbf{8}$ & $\mathbf{9}$ & $\mathbf{1 0}$ \\
\hline \hline$n$ & 1.4 & 1.5 & 1.33 & 1.4 & 1.41 & 1.35 & 1.36 & 1.37 & 1.5 & 1.3 \\
\hline
\end{tabular}

Table 3: Co-contraction factor for each subject

\begin{tabular}{|c||c|c|c||c|c|c|}
\hline & \multicolumn{3}{|c|}{$k_{\text {extension }}$} & \multicolumn{3}{c|}{$k_{\text {flexion }}$} \\
\hline$i$ & Minimum & Maximum & Average & Minimum & Maximum & Average \\
\hline 1 & -0.1212 & 0.0921 & 0.0116 & 0.0738 & 0.5338 & 0.1995 \\
2 & 0.2345 & 0.6085 & 0.3647 & 0.1558 & 0.3661 & 0.2263 \\
3 & 0.5258 & 1.0287 & 0.8084 & 0.3498 & 0.6798 & 0.4761 \\
4 & 1.5477 & 3.1993 & 2.0990 & 1.4302 & 2.4185 & 1.8250 \\
5 & 0.3631 & 0.8961 & 0.5853 & 0.4400 & 0.8827 & 0.6172 \\
6 & 0.0722 & 0.5578 & 0.2367 & 0.2140 & 0.7959 & 0.4289 \\
7 & 0.0237 & 0.0991 & 0.0634 & 0.0036 & 0.1009 & 0.0436 \\
8 & 0.1861 & 0.5061 & 0.3281 & 0.2018 & 1.0673 & 0.6136 \\
9 & 0.3571 & 1.2996 & 0.7610 & 0.1424 & 0.8340 & 0.4853 \\
10 & 0.3930 & 0.4865 & 0.4398 & 0.2847 & 0.5810 & 0.4329 \\
\hline
\end{tabular}

Table 4: Experimentally calculated values of ' $k$ ' for flexion and extension motion

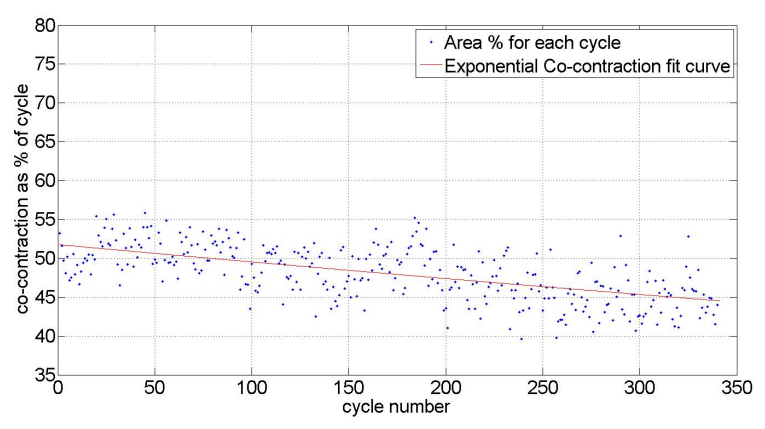

Figure 9: Curve fit of co-contraction area, subject 1

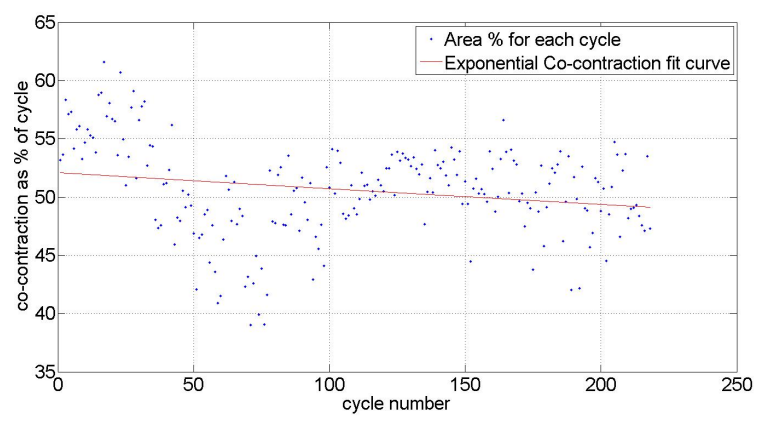

Figure 10: Curve fit of co-contraction area, subject 2 


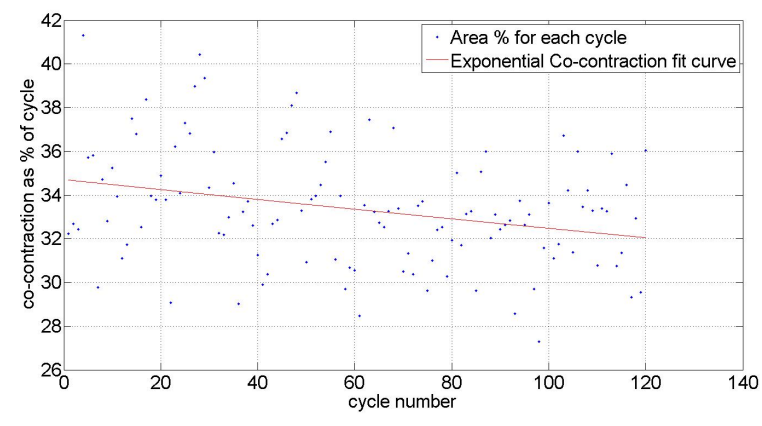

Figure 11: Curve fit of co-contraction area, subject 3

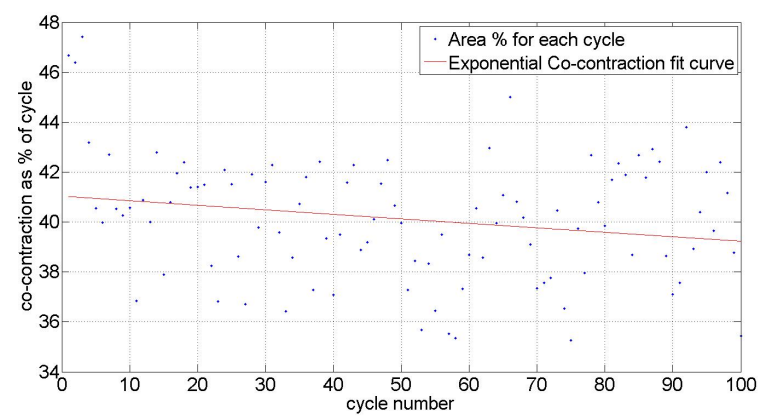

Figure 12: Curve fit of co-contraction area, subject 4

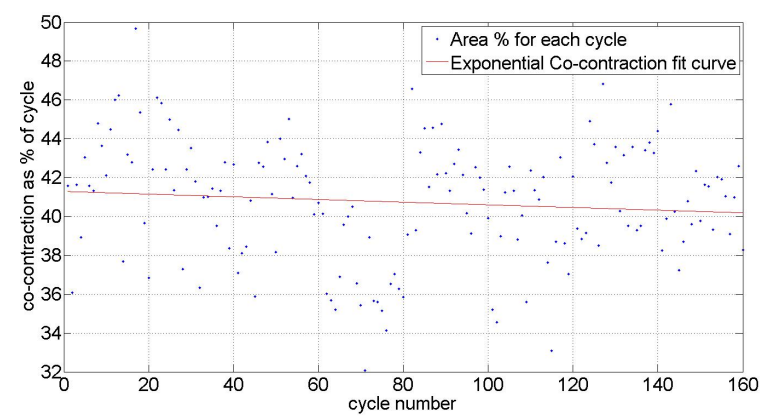

Figure 13: Curve fit of co-contraction area, subject 5

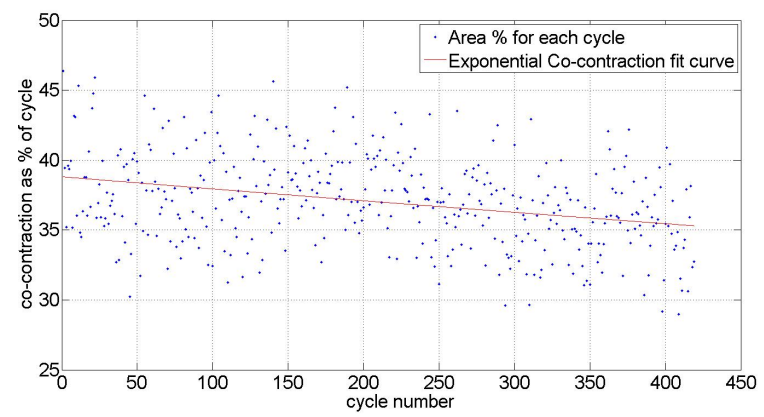

Figure 14: Curve fit of co-contraction area, subject 6

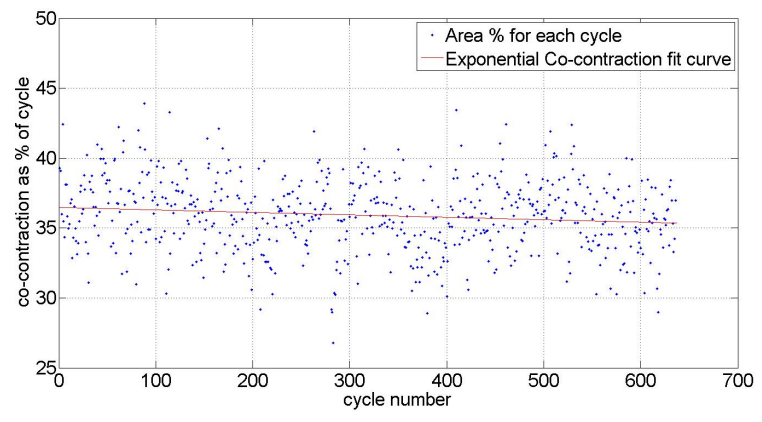

Figure 15: Curve fit of co-contraction area, subject 7

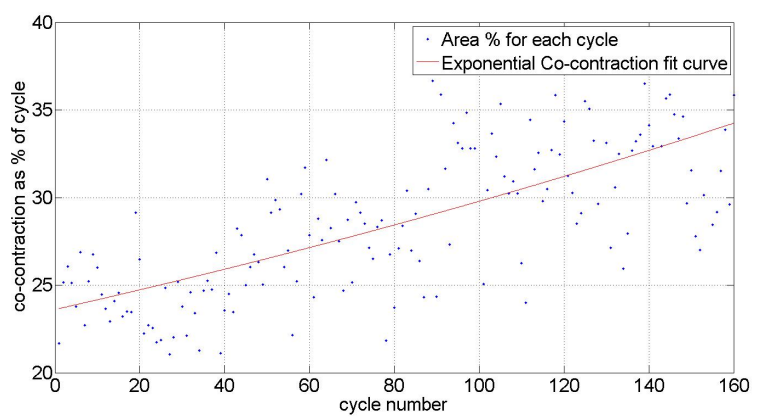

Figure 16: Curve fit of co-contraction area, subject 8

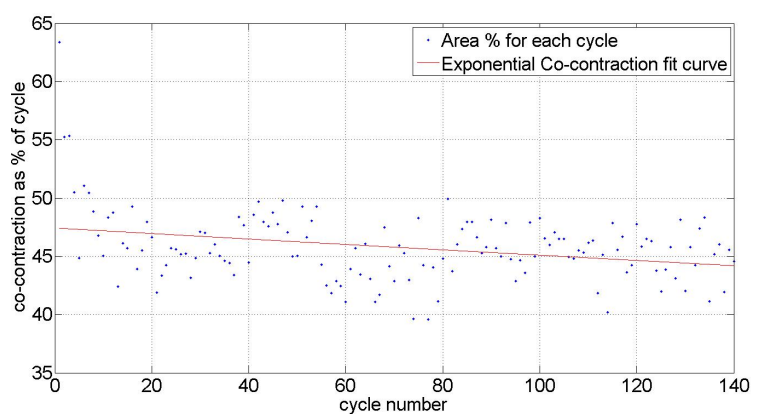

Figure 17: Curve fit of co-contraction area, subject 9

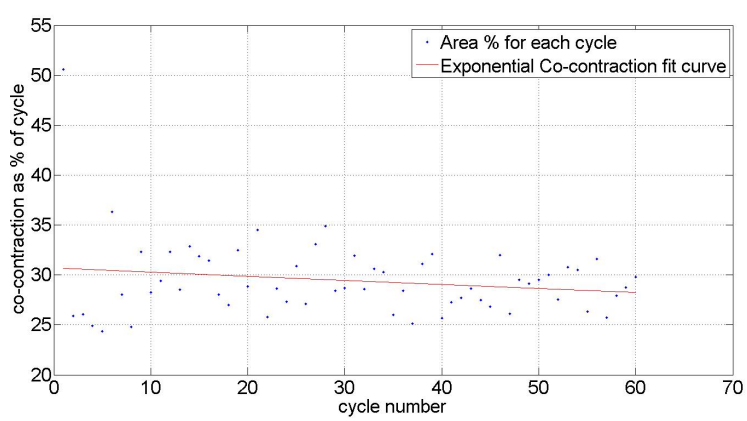

Figure 18: Curve fit of co-contraction area, subject 10 


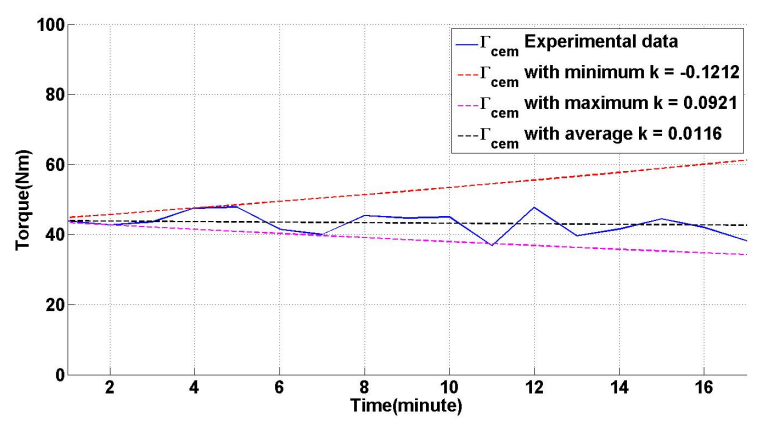

Figure 19: $\Gamma_{c e m}$ evaluation for extension phase, subject 1

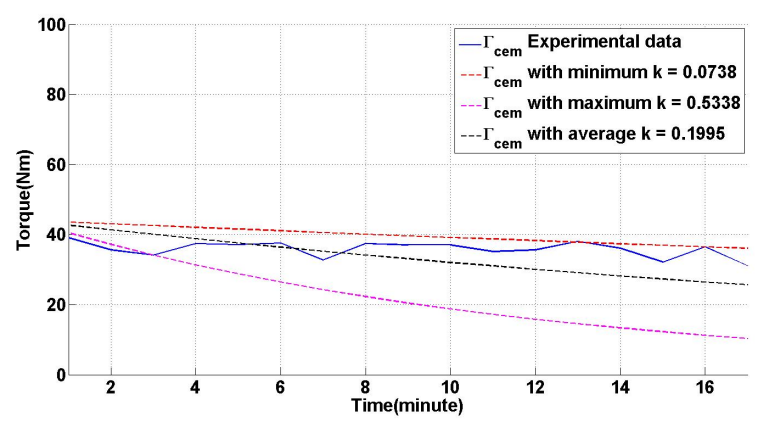

Figure 20: $\Gamma_{c e m}$ evaluation for flexion phase, subject 1

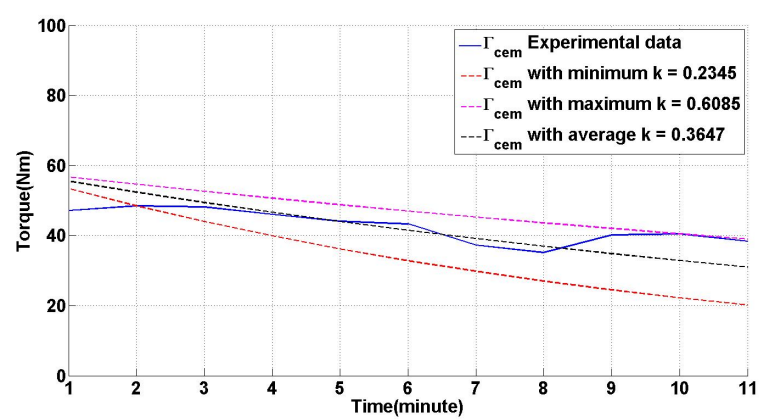

Figure 21: $\Gamma_{c e m}$ evaluation for extension phase, subject 2

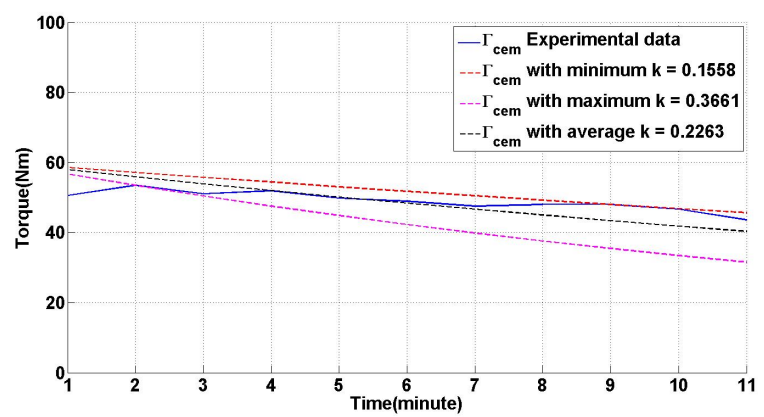

Figure 22: $\Gamma_{c e m}$ evaluation for flexion phase, subject 2

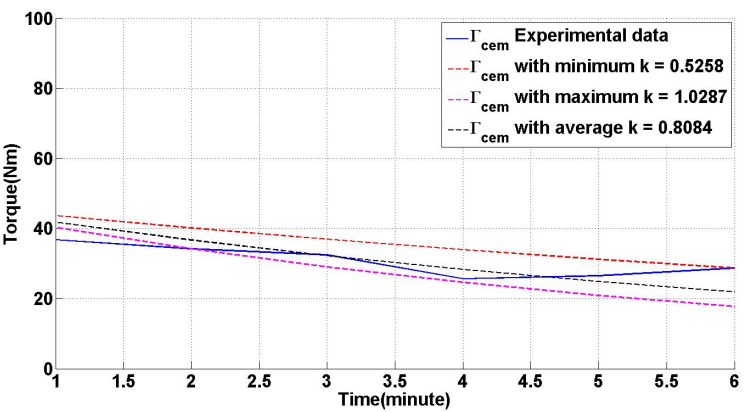

Figure 23: $\Gamma_{c e m}$ evaluation for extension phase, subject 3

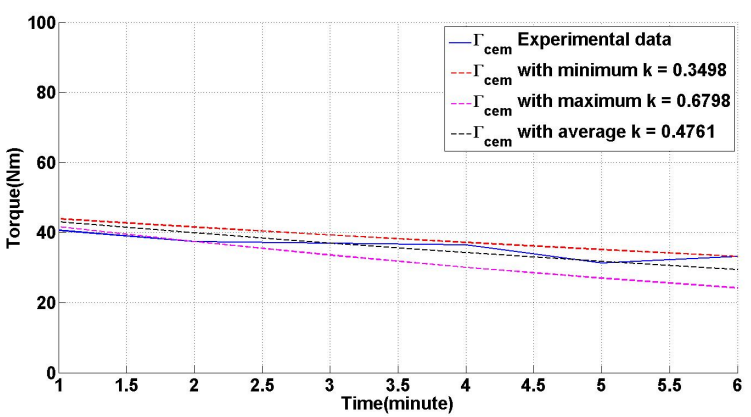

Figure 24: $\Gamma_{c e m}$ evaluation for flexion phase, subject 3

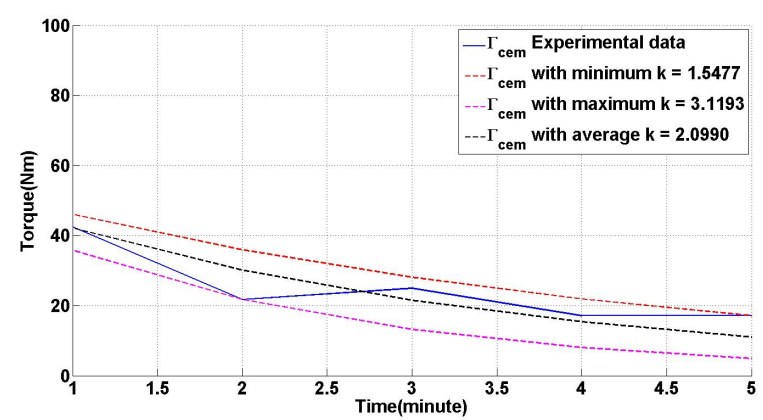

Figure 25: $\Gamma_{c e m}$ evaluation for extension phase, subject 4

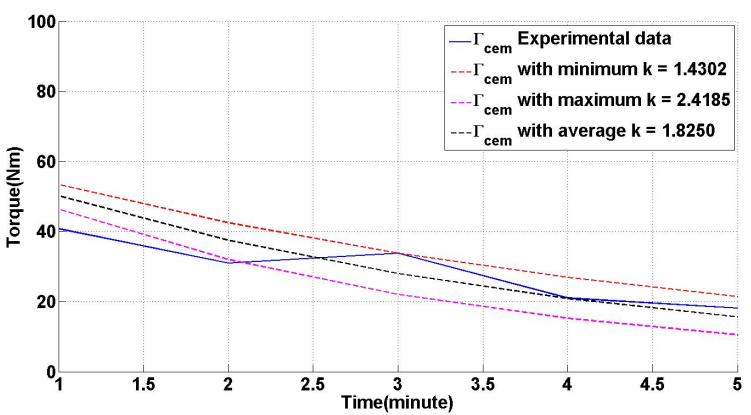

Figure 26: $\Gamma_{c e m}$ evaluation for flexion phase, subject 4 


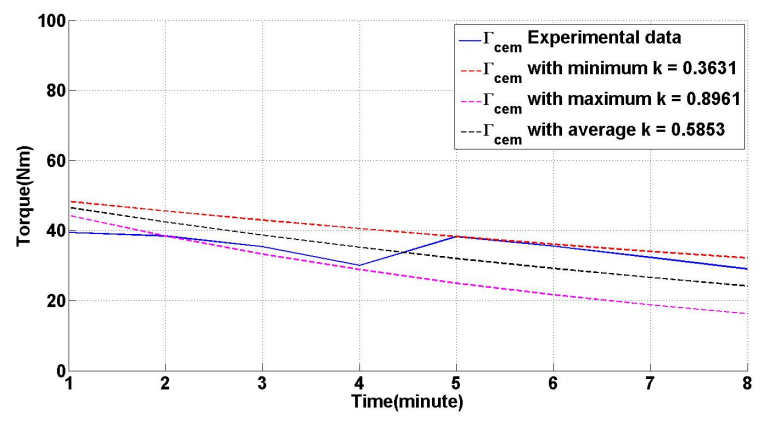

Figure 27: $\Gamma_{c e m}$ evaluation for extension phase, subject 5

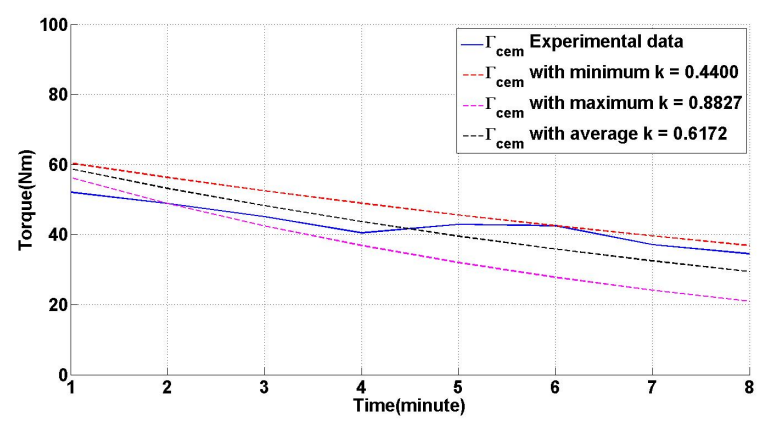

Figure 28: $\Gamma_{c e m}$ evaluation for flexion phase, subject 5

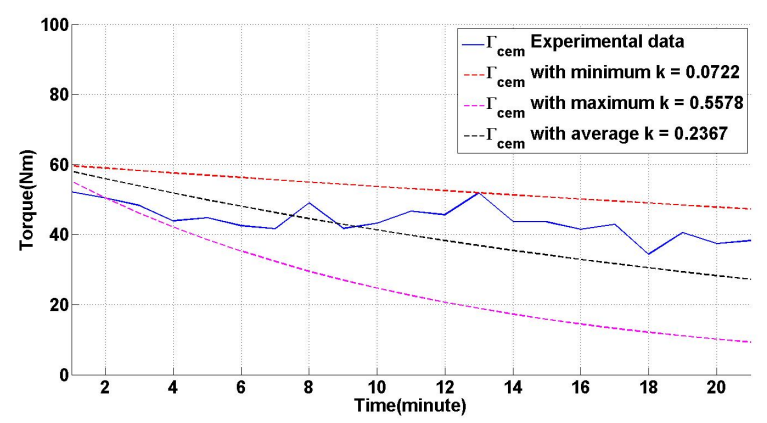

Figure 29: $\Gamma_{c e m}$ evaluation for extension phase, subject 6

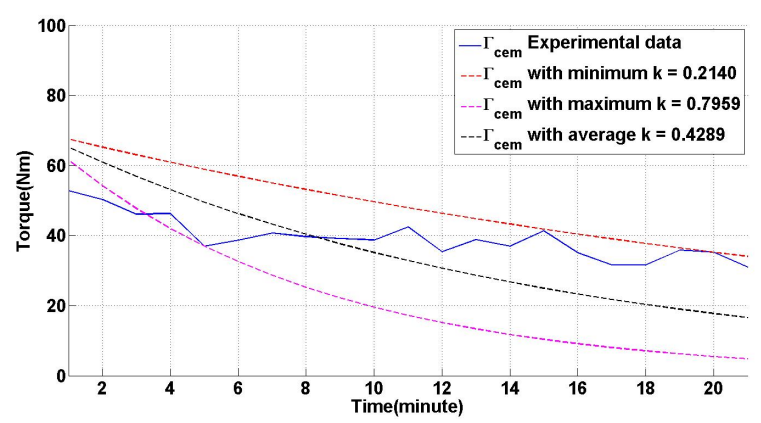

Figure 30: $\Gamma_{c e m}$ evaluation for flexion phase, subject 6

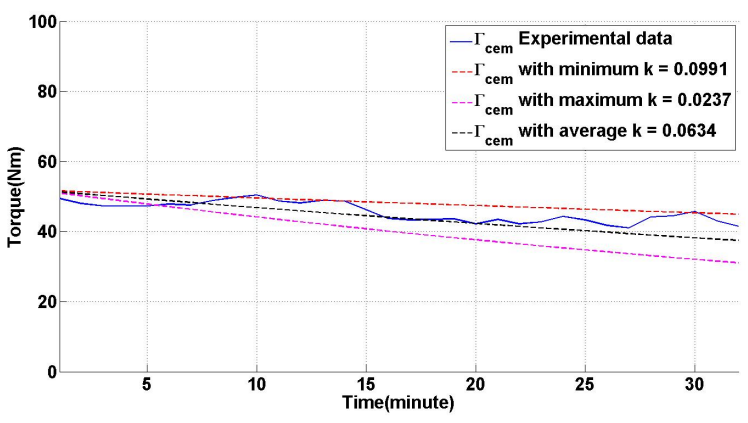

Figure 31: $\Gamma_{c e m}$ evaluation for extension phase, subject 7

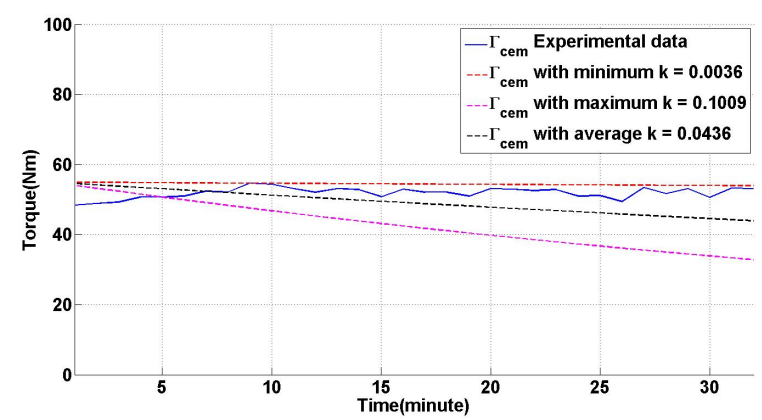

Figure 32: $\Gamma_{\text {cem }}$ evaluation for flexion phase, subject 7

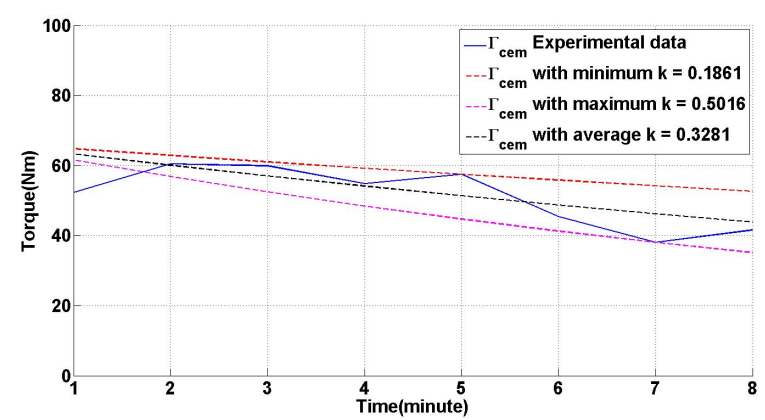

Figure 33: $\Gamma_{c e m}$ evaluation for extension phase, subject 8

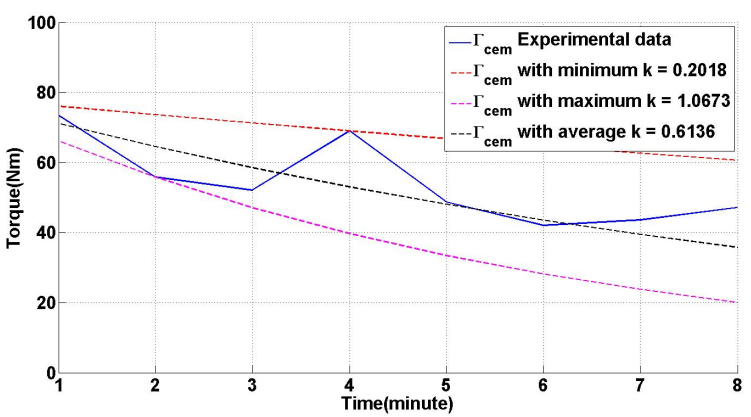

Figure 34: $\Gamma_{c e m}$ evaluation for flexion phase, subject 8 


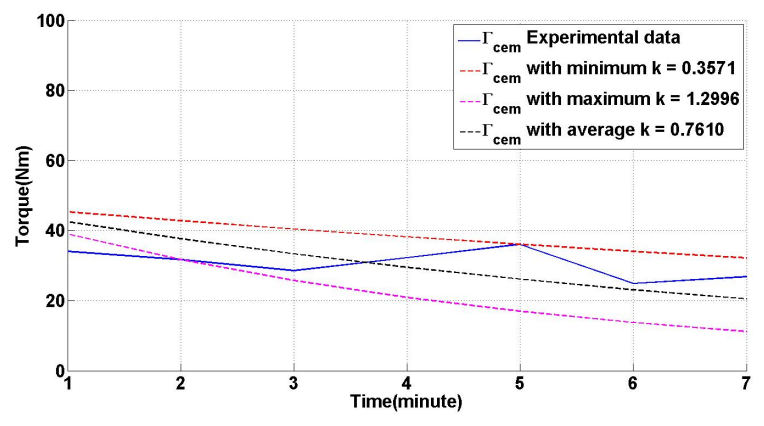

Figure 35: $\Gamma_{c e m}$ evaluation for extension phase, subject 9

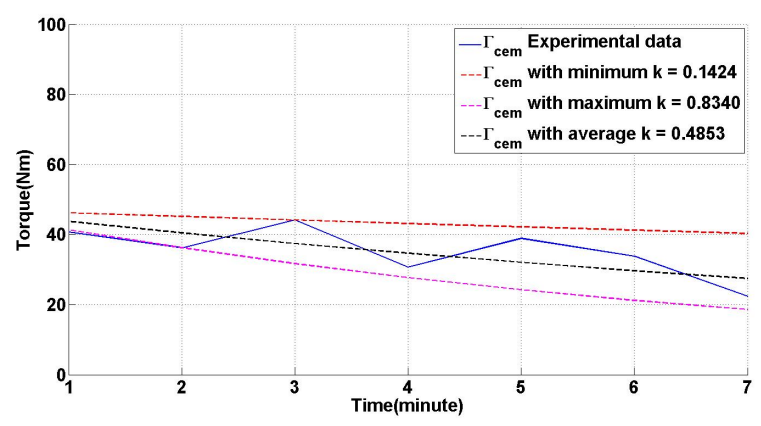

Figure 36: $\Gamma_{c e m}$ evaluation for flexion phase, subject 9

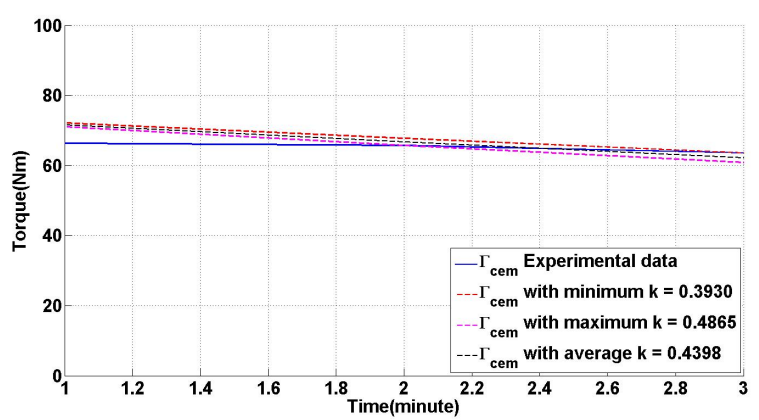

Figure 37: $\Gamma_{c e m}$ evaluation for extension phase, subject 10

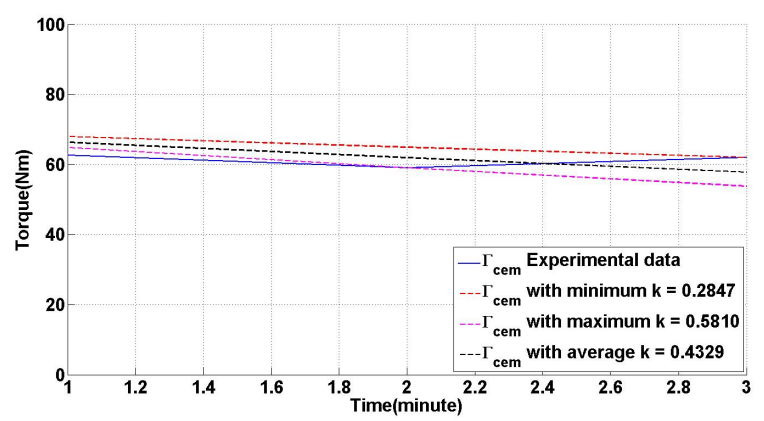

Figure 38: $\Gamma_{c e m}$ evaluation for flexion phase, subject 10

\section{DMET Analysis}

The dynamic maximum endurance time was calculated for each subject according to their respective fatigue rate values. The results are given in Tab. 5 The fatigue rate for the flexion and extension phases are different for each subject. We have selected the maximum average value of $k$ from both the phases using Eq. 3. The reason behind the particular selection of values of $k$ is to make model more safe and hence analysis on the basis of that value. More the fatigue rate we choose for work design with Seth's model safer will the endurance time for the subject. In DMET calculations, ' $d$ ' represents the dynamic factor as mentioned before in Eq. 4 ranging between 0.1 to 1 . The larger the value of $d$ represent more static conditions. A smaller value of $d$ represent more dynamic model.

The comparison between the DMET calculated for the same subject with R. Ma's model and MET calculated by L. Ma's model has been done. The DMET comparison is shown in Tab. 5. The DMET is calculated using Eq. 4 for proposed model. MET calculation for L. Ma is done by the same equation with $n=1$ and $d=1$ because this model is for static conditions and without co-contraction. The DMET calculation for $\mathrm{R}$. Ma is also done by the same equation with $n=1$ and $d=0.5$ because there is no co-contraction included and dynamic factor is for medium dynamic motion. For Seth's model, the DMET is calculated with the parameters, $n=1.38$ and $d=0.5$. The percentage difference between the DMET calculated from Seth's model and experiment test duration is also presented in Tab. 5. The DMET is calculated for each subject on the basis of their maximum fatigue rate ' $k$ ' so that the DMET calculated can be safer to subjects. According to fatigue experiment protocol for each subject, the load was $20 \%$ of MVC. The values of load for each subject corresponding to their maximum MVC values are also presented in table 5.

The DMET is also predicted for Seth's model keeping the value of co-contraction factor $n=1.38$ at the value of $d=0.5$. Fig. 39 represents the DMET for subjects with respect to the value of load $f_{M V C}$, which is the ratio of external load to the maximum capacity or MVC of a subject, see Tab.dmettable2 for the values of the load for each subject. We can observe in Fig. 39 that DMET for Seth's model (red line) is less than R. Ma's model (green line) and more than L. Ma's model (blue line).

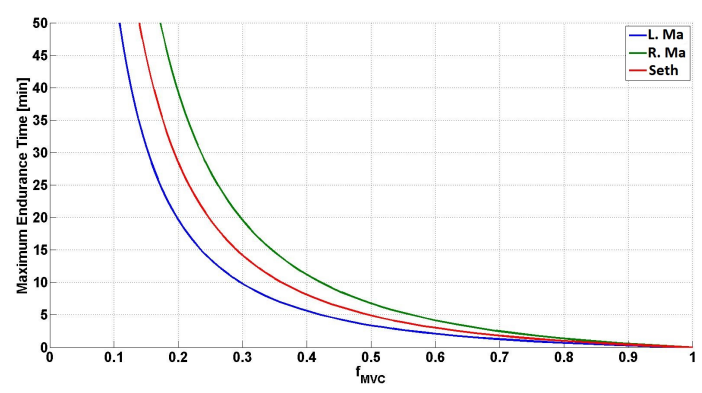

Figure 39: DMET prediction at $d=0.5, k=0.41$ 
The International Journal of Virtual Reality , 2016, 16(1): pp22-pp32

\begin{tabular}{|c|c|c|c|c|c|c|c|c|}
\hline $\begin{array}{c}\text { Subject } \\
\text { Number }\end{array}$ & $\begin{array}{c}\text { Test Duration } \\
\text { (Minutes) }\end{array}$ & $\begin{array}{c}\text { MET-L. Ma } \\
\text { (Minutes) }\end{array}$ & $\begin{array}{c}\text { DMET-R. Ma } \\
\text { (Minutes) }\end{array}$ & $\begin{array}{c}\text { DMET-Seth } \\
\text { (Minutes) }\end{array}$ & \% difference & $\begin{array}{c}\text { K } \\
\text { Value }\end{array}$ & $\begin{array}{c}\text { Load } \\
\text { (N.m) }\end{array}$ & $\begin{array}{c}\text { MVC } \\
\text { (N.m) }\end{array}$ \\
\hline \hline 1 & 17 & 11.2 & 22.4 & 17.8 & 4.5 & 0.3043 & 8.8 & 44.02 \\
2 & 11 & 9.69 & 19.38 & 14.35 & 23.3 & 0.3606 & 11.6 & 58.58 \\
3 & 6 & 5.73 & 11.46 & 8.5 & 29.4 & 0.6096 & 9.5 & 47.5 \\
4 & 5 & 3.46 & 6.920 & 5.12 & 2.3 & 1.08 & 11.8 & 59.01 \\
5 & 8 & 6.59 & 13.18 & 9.76 & 18.03 & 0.53 & 10.2 & 51.1 \\
6 & 21 & 14.5 & 29.12 & 21.57 & 2.6 & 0.25 & 12.06 & 60.32 \\
7 & 32 & 24.96 & 49.92 & 36.98 & 13.43 & 0.14 & 10.36 & 51.8 \\
8 & 8 & 5.8 & 11.64 & 8.62 & 7.19 & 0.61 & 13.34 & 66.7 \\
9 & 7 & 4.9 & 9.98 & 7.4 & 5.4 & 0.7 & 9.5 & 47.9 \\
10 & 3 & 10.2 & 20.5 & 15.2 & 80.2 & 0.34 & 15.36 & 76.82 \\
\hline
\end{tabular}

Table 5: Maximum Endurance Time Comparison

We can see that the DMET calculated for each subject is more than the experimented value. It is because DMET is the maximum limit of any human and we did the experiment for each subject up to comfortable exhaustion level. Comfortable exhaustion level means the level at which the subjects want to stop the experiment because of fatigue. It may be possible in this cases that the subjects do not reach their maximum limit but they stop the test, for example, subject number 10, we can see in Tab. 5 that he stopped the test after 3 minutes, after completing 60 cycles but the maximum endurance time is much larger than the experiment duration. For subject 10 the percentage difference between the DMET calculated by Seth's model and experiment duration is $80.2 \%$, which is much higher in comparison to other subjects. L. Ma's model is a static model, that is why maximum endurance time calculated is less than the experimented value. R. Ma's model gives more DMET for each subject in comparison to Seth's DMET model which is much closer to the experimental values. The DMET calculated by Seth's model is $25.9 \%$ less than the DMET calculated by R. Ma's model. The DMET calculated for Seth's model is less because we have introduced the co-contraction factor into the model. This gives more approximate value to the experimental data. So the work design according to this model will be safer in comparison to R. Ma's Model, L. Ma's model is for static posture hence, it may not be real to compare for dynamic situations.

\section{Conclusions}

The proposed model for dynamic muscle fatigue includes the co-contraction parameter, unlike in any other existing model according to the author's knowledge. The results and analysis of the experimental data validate best of the assumptions made for the proposed model. EMG analysis along with MVC helps to understand the muscle activities, also it justifies the significance of the co-contraction parameter in the proposed dynamic muscle fatigue model (Seth's model). The experimental data also helps in validating the new dynamic muscle fatigue model. The co-contraction factor allowed reducing DMET. The DMET model validation shows that there is a reduction of endurance time in comparison with R. Ma model and in static cases with L. Ma model.
The DMET comparison shows that there is $25.9 \%$ reduction in the maximum endurance time in Seth's model as compared to R. Ma's model. When we compare the DMET at dynamic variation factor, $\mathrm{d}=0.5$, it shows that the time taken by different subjects to complete the fatigue protocols during the experiment are near to the DMET values calculated for maximum values of $k$ and closer to Seth's model. It shows that Seth's DMET model is more safer and better for dynamic condition in comparison to other models.

\section{REFERENCES}

[Ahrache, Imbeau, and Farbos 2006] Ahrache, K. E.; Imbeau, D.; and Farbos, B. 2006. Percentile values for determining maximum endurance times for static muscular work. International Journal of Industrial Ergonomics 26:99-108.

[Chaffin, Andersson, and Martin. 1999] Chaffin, D. B.; Andersson, G. B. J.; and Martin., B. J. 1999. Occupational Biomechanics. Wiley - Interscience, third edition.

[Ding, Wexler, and Binder-Macleod 2003] Ding, J.; Wexler, A. S.; and Binder-Macleod, S. A. 2003. Mathematical models for fatigue minimization during functional electrical simulation. journal of electromyograpgy and kinesiology 13:575-588.

[Doguet and Jubeau 2014] Doguet, V., and Jubeau, M. 2014. Reliability of h-reflex in vastus lateralis and vastus medialis muscles during passive and active isometric conditions. European Journal of Applied Physiology 114(12):2509-19.

[Freund and Takala 2001] Freund, J., and Takala, E.-P. 2001. A dynamic model of the forearm including fatigue. Journal of Biomechanics 34:597-605.

[Guvel et al. 2011] Guvel, A.; Boyas, S.; Guihard, V.; Cornu, C.; Hug, F.; and Nordez, A. 2011. Thigh muscle activities in elite rowers during on-water rowing. International Journal of Sports Medicine 32:109-116.

[Hill 1938] Hill, A. 1938. The heat of shortening and dynamic constant of muscle. Proceedings of the Royal Society of Biological Sciences 126:135-195.

[Huppe, Muller, and Raspe 2006] Huppe, A.; Muller, K.; and Raspe, H. 2006. Is the occurrence of back pain in ger- 
many decreasing? two regional postal surveys a decade apart. European Journal of Public Health 17:318-322.

[Liu, Brown, and Yue 2002] Liu, J. Z.; Brown, R. W.; and Yue, G. H. 2002. A dynamical model of muscle activation, fatigue and recovery. Biophysical Journal 82:23442359 .

[Ma et al. 2008] Ma, L.; Chablat, D.; Bennis, F.; and Zhang, W. 2008. A new muscle fatigue and recovery model and its ergonomics application in human simulation. Virtual and Physical Prototyping 5:123-137.

[Ma et al. 2009] Ma, L.; Chablat, D.; Bennis, F.; and Zhang, W. 2009. A new simple dynamic muscle fatigue model and its validation. International Journal of Industrial Ergonomics 39(1):211-220.

[Ma et al. 2011] Ma, R.; Chablat, D.; Bennis, F.; and Ma, L. 2011. A framework of motion capture system based human behaviours simulation for ergonomic analysis. In HCI International 2011, 9-14 July, Hilton Orlando Bonnet Creek, Orlando, Florida, USA.

[Ma, Chablat, and Bennis 2012a] Ma, R.; Chablat, D.; and Bennis, F. 2012a. Human muscle fatigue model in dynamic motions. Latest Advances in Robot Kinematics $0: 349-356$.

[Ma, Chablat, and Bennis 2012b] Ma, R.; Chablat, D.; and Bennis, F. 2012b. A new approach to muscle fatigue evaluation for push/pull task. In 19th CISM-IFToMM Symposium on Robot Design, Dynamics, and Control, Paris, France, 1-8.

[Ma 2012] Ma, R. 2012. Modélisation de la fatigue musculaire dynamique et son application pour l'analyse ergonomique. Ph.D. Dissertation, IRCCyN, Ecole Centrale de Nantes.

[Missenard, Mottet, and Perrey 2008] Missenard, O.; Mottet, D.; and Perrey, S. 2008. Muscular fatigue increases signal-dependent noise during isometric force production. Neuroscience Letters 437:154-157.

[Nur, Dawal, and Dahari 2014] Nur, N. M.; Dawal, S. Z. M.; and Dahari, M. 2014. The prevelence of work related musculosceletal disorders among workers performing industrial repetitive tasks in the automotive manufacturing companies. In Proceedings of the 2014 International conference on inductrial engineering and operations management, Bali, Indonesia.

[Punnett and Wegman 2004] Punnett, L., and Wegman, D. H. 2004. Work related musculoskeletal disorders: the epidemiologic evidence and the debate. Journal of Electromyography and Kinesiology 14:13-23.

[Seth et al. 2016] Seth, D.; Chablat, D.; Bennis, F.; Sakka, S.; Jubeau, M.; and Nordez, A. 2016. New dynamic muscle fatigue model to limit musculo-skeletal disorder. In 2016 Virtual Reality International Conference, Laval, France.

[Silva, Pereira, and Martins 2011] Silva, M. T.; Pereira, A. F.; and Martins, J. M. 2011. An efficient muscle fa- tigue model for forward and inverse dynamic analysis of human movements. Procedia IUTAM 2:262-274.

[Syuzev, Gouskov, and Galiamova 2010] Syuzev, V. V.; Gouskov, A. M.; and Galiamova, E. V. 2010. Human skeletal muscle - mechanical and mathematical models. ICABB-2010, Venice, Italy 0.

[Vøllestad 1997] Vøllestad, N. K. 1997. Measurement of human muscle fatigue. Journal of Neuroscience Methods 74:219-227.

[World-Health-Organization 2003] World-HealthOrganization. 2003. Preventing musculoskeletal disorders in the workplace. Protecting workers 'Health series; no. 5', Geneva 27, Switzeland. ISBN 924159053 $\mathrm{X}$.

[Xia and Lawa 2008] Xia, T., and Lawa, L. A. F. 2008. A theoretical approach for modeling peripheral muscle fatigue and recovery. Journal of Biomechanics 41:30463052 . 